\title{
Elevated Serum Levels of the Antiapoptotic Protein Decoy-Receptor 3 Are Associated with Advanced Liver Disease
}

\author{
Giorgos Bamias, ${ }^{1}$ Michalis Gizis, ${ }^{1}$ Ioanna Delladetsima, ${ }^{2}$ \\ Eyfrosyni Laoudi, ${ }^{1}$ Spyros I. Siakavellas, ${ }^{1}$ Ioannis Koutsounas, ${ }^{1}$ \\ Garyfallia Kaltsa, ${ }^{1}$ John Vlachogiannakos, ${ }^{1}$ Irene Vafiadis-Zouboulis, ${ }^{1}$ \\ George L. Daikos, ${ }^{3}$ George V. Papatheodoridis, ${ }^{1}$ and Spiros D. Ladas ${ }^{1}$ \\ ${ }^{1}$ Academic Department of Gastroenterology, Medical School of National and Kapodistrian University of Athens, \\ Laiko General Hospital, Athens 11527, Greece \\ ${ }^{2}$ First Department of Pathology, Medical School of National and Kapodistrian University of Athens, Laiko General Hospital, \\ Athens 11527, Greece \\ ${ }^{3}$ Infectious Diseases Research Laboratory, Medical School of National and Kapodistrian University of Athens, \\ Laiko General Hospital, Athens 11527, Greece \\ Correspondence should be addressed to Giorgos Bamias; gbamias@gmail.com
}

Received 3 February 2016; Revised 28 June 2016; Accepted 17 July 2016

Academic Editor: Aldo J. Montano-Loza

Copyright (C) 2016 Giorgos Bamias et al. This is an open access article distributed under the Creative Commons Attribution License, which permits unrestricted use, distribution, and reproduction in any medium, provided the original work is properly cited.

Background. Decoy-receptor 3 (DcR3) exerts antiapoptotic and immunomodulatory function and is overexpressed in neoplastic and inflammatory conditions. Serum DcR3 (sDcR3) levels during the chronic hepatitis/cirrhosis/hepatocellular carcinoma (HCC) sequence have not been explored. Objective. To assess the levels and significance of sDcR3 protein in various stages of chronic liver disease. Methods. We compared sDcR3 levels between healthy controls and patients with chronic viral hepatitis (CVH), decompensated cirrhosis (DC), and HCC. Correlations between sDcR3 levels and various patient- and disease-related factors were analyzed. Results. sDcR3 levels were significantly higher in patients with CVH than in controls $(P<0.01)$. sDcR3 levels were elevated in DC and HCC, being significantly higher compared not only to controls $(P<0.001$ for both) but to CVH patients as well $(P<0.001$ for both). In addition, DcR3 protein was detected in large quantities in the ascitic fluid of cirrhotics. In patients with $\mathrm{CVH}$, sDcR3 significantly correlated to fibrosis severity, as estimated by Ishak score $(P=0.019)$ or by liver stiffness measured with elastography (Spearman $r=0.698, P<0.001$ ). In cirrhotic patients, significant positive correlations were observed between sDcR3 levels and markers of severity of hepatic impairment, including MELD score $(r=0.653, P<0.001)$. Conclusions. Circulating levels of DcR3 are elevated during chronic liver disease and correlate with severity of liver damage. sDcR3 may serve as marker for liver fibrosis severity and progression to end-stage liver disease.

\section{Introduction}

Hepatic carcinogenesis is a multistep process, which develops through sequential stages [1]. Hepatocellular carcinoma (HCC) develops mainly on cirrhotic livers through malignant transformation of regenerative nodules. Cirrhosis is usually the end result of chronic necroinflammatory activity (chronic hepatitis) which causes repeated cycles of hepatic injury and repair [2]. The critical biological event is the development of fibrosis, which eventually leads to distortion of liver architecture and deterioration of hepatic function. Discrimination between different stages is of great importance for the individual patient, as prognostic significance and therapeutic implications usually differ significantly in each stage. 
Therefore, the identification of markers which can be easily detected and are associated with the severity of fibrosis would be of great clinical application.

Decoy-receptor 3 (DcR3) belongs to the tumor necrosis factor receptor superfamily of proteins (TNFRSF). It is capable of binding to three different TNF-like ligands, FasL, TL1A, and LIGHT, thus blocking their interaction with their functional receptors (Fas, DR3, and $\operatorname{LTR} \beta$, resp.) $[3,4]$. Through this inhibitory function, DcR3 exerts antiapoptotic and immunomodulatory properties. Accordingly, it was proposed that DcR3 may promote tumorigenesis not only by directly inhibiting tumor cell apoptosis but also by ameliorating cytolytic T-cell responses and recruitment of acute inflammatory cells, as well as by promoting angiogenesis. In addition, DcR3 has also been shown to block proinflammatory signals mediated by the aforementioned TNF/TNFRSF ligand-receptor pairs. In line with these functional data, expression studies have clearly demonstrated overexpression of DcR3 in a variety of inflammatory and neoplastic conditions [5-8]. A distinctive property of DcR3 is that it lacks a transmembrane region and exists only as soluble protein, which can be accurately determined in biological fluids [9]. DcR3 is not detected or exists in low levels in the peripheral blood of healthy individuals; but it is secreted in the systemic circulation, often reaching substantial concentrations, during several inflammatory and neoplastic conditions [7, 10-13].

The dual biological functionality of DcR3 (i.e., immunoregulatory and antiapoptotic) raises the possibility that it may be involved in the chronic hepatitis/cirrhosis/HCC sequence, as inflammation takes place in the initial and malignant transformation in the late stage. Previous studies have focused on the association between DcR3 and HCC, reporting increased local and systemic expression of DcR3 in patients with HCC [14-16]. On the other hand, a systematic evaluation of the levels of circulating DcR3 protein during the progression from chronic hepatitis to cirrhosis and, eventually, to HCC has not been explored so far. In addition, the potential prognostic significance of elevated serum DcR3 protein in patients with chronic liver disease is unknown at present.

We undertook the present study to comparatively evaluate the sDcR3 protein levels in patient populations at various stages of chronic liver disease (chronic hepatitis versus cirrhosis versus HCC). We report elevated levels of circulating DcR3 in these conditions, independently of the cause of liver disease, and provide evidence for a critical association between liver fibrosis severity and increased sDCR3. We also demonstrate that sDcR3 levels positively correlate with adverse disease outcomes and may therefore serve as a poor prognostic factor in patients with chronic liver disease.

\section{Materials and Methods}

2.1. Patients. The study population consisted of 125 consecutive patients with chronic liver disease, who were followed up at our department between January 2009 and December 2011. Chronic viral hepatitis $(\mathrm{CVH})$ was diagnosed in patients without any sign of decompensated cirrhosis (DC) or HCC, who had positive hepatitis B virus (HBV) surface antigen or antibodies to hepatitis $\mathrm{C}$ virus (HCV) for at least 6 months and detectable viremia of $\mathrm{HBV}$ or $\mathrm{HCV}$, respectively. $\mathrm{DC}$ was diagnosed in patients with any type of chronic liver disease who had a history of at least one sign of liver decompensation (ascites, variceal bleeding, hepatic encephalopathy, and nonobstructive jaundice). The diagnosis of HCC was based on histological and/or radiological findings according to international criteria [17].

Demographic and clinical information was obtained from the patients' medical records. Laboratory parameters were recorded concomitantly with the determination of sDcR3 levels. In patients with $\mathrm{CVH}$ who had undergone a liver biopsy, the severity of histological lesions for both necroinflammatory activity (grade) and fibrosis (stage) was assessed according to the classification of Ishak et al. [18]. All biopsies were considered to be adequate as they fulfilled the minimum requirements for adequate liver specimens (at least 6 portal tracts and length $\geq 2 \mathrm{~cm}$ ). The severity of fibrosis was also assessed by shear wave elastography (SuperSonic Imagine, SA, Aix-en-Provence, France) and expressed as liver stiffness values in $\mathrm{kPa}$ in a subgroup of chronic liver disease patients. The severity of liver disease was assessed by the Model for End-Stage Liver Disease (MELD) in patients with DC. Further important divisions of patients' subgroups (such as treatment-naive patients in the $\mathrm{CVH}$ group or classification according to the presence of fibrosis) were identified and used in our analyses. In particular, patients were considered to have severe fibrosis if they had histological fibrosis stage of $\geq 4$ at liver biopsy or liver stiffness value of $>10 \mathrm{kPa}$ at elastography. In addition, patients were considered to have advanced liver disease if they had severe fibrosis according to the previous definition or clinical signs of decompensated cirrhosis.

Age- and sex-matched healthy blood donors (controls) were planned to be included at a ratio of 1 per 2 cases. However, it was eventually impossible to find appropriate HC for all cases because of the old age of some patients. Thus, 48 healthy controls were eventually included. The study was approved by the local ethics committee and was in line with the Helsinki Declaration of 1975, as revised in 2008. Informed consent was obtained from all patients and controls.

2.2. Sample Collection. Venous blood samples were collected from patients and controls and centrifuged at $3000 \mathrm{rpm}$. In cases with elastography, a venous blood sample was collected just prior to the test. In cases with biopsy, a venous blood sample was collected on the day of admission for liver biopsy. Sera were separated, aliquoted, and stored at $-80^{\circ} \mathrm{C}$ until being used. Ascitic fluid was also collected from a subgroup of cirrhotic patients who were admitted for therapeutic paracentesis. Ascitic fluid was collected and centrifuged at $3000 \mathrm{rpm}$. The supernatant was collected and stored immediately at $-80^{\circ} \mathrm{C}$.

2.3. Measurement of Concentration of DcR3 by ELISA. The levels of DcR3 in serum and ascites were determined using the human DcR3 DuoSet ELISA Development Kit (R\&D 
TABLE 1: Clinical and demographic characteristics of patients.

\begin{tabular}{|c|c|c|c|}
\hline & $\begin{array}{l}\text { Chronic hepatitis } \\
\quad(n=58)\end{array}$ & $\begin{array}{l}\text { Cirrhosis } \\
(n=46)\end{array}$ & $\begin{array}{c}\text { HCC } \\
(n=21)\end{array}$ \\
\hline Age, years (mean \pm SD (range)) & $43.9 \pm 12.7(25-68)$ & $64.9 \pm 11.0(46-89)$ & $61.3 \pm 9.9(50-77)$ \\
\hline Male sex (\%) & $40(69 \%)$ & $35(76.1 \%)$ & $20(95.2 \%)$ \\
\hline \multicolumn{4}{|l|}{ Cause of liver disease $(n, \%)$} \\
\hline HBV infection & $28(48.3 \%)$ & $11(23.9 \%)$ & $9(42.9 \%)$ \\
\hline HCV infection & $30(51.7 \%)$ & $14(30.4 \%)$ & $5(23.8 \%)$ \\
\hline Alcohol & & $14(30.4 \%)$ & \\
\hline Unknown & & $7(15.2 \%)$ & $7(33.3 \%)$ \\
\hline \multicolumn{4}{|l|}{ Histological grade $(n=34)$} \\
\hline Mild necroinflammation (1-6) & $23(67.6 \%)$ & NA & NA \\
\hline Moderate (7-12) & $9(26.5 \%)$ & NA & NA \\
\hline Severe (13-18) & $2(7.9 \%)$ & NA & NA \\
\hline \multicolumn{4}{|l|}{ Histological stage $(n=34)$} \\
\hline No fibrosis $(0)$ & $14(41.2 \%)$ & NA & NA \\
\hline Minimal (1-2) & $9(26.5 \%)$ & NA & NA \\
\hline Moderate (3-4) & $7(20.5 \%)$ & NA & NA \\
\hline Severe (5-6) & $4(11.8 \%)$ & NA & NA \\
\hline MELD score (mean \pm SD (range)) & NA & $15.89 \pm 9.27(3-43)$ & \\
\hline Liver stiffness $(\mathrm{kPa})($ mean $\pm \mathrm{SD}$ (range) $)$ & $7.91 \pm 1.68(5-11)$ & $\begin{array}{l}33.11 \pm 13.22 \\
(22-66)\end{array}$ & NA \\
\hline
\end{tabular}

NA: not applicable.

Systems, MN), according to the manufacturer's instructions with minor modifications. Briefly, $4.0 \mu \mathrm{g} / \mathrm{mL}$ of mouse antihuman anti-DcR3 antibody was incubated overnight in flatbottomed microplates. Nonspecific binding was blocked with $1 \%$ BSA in PBS. Following thorough washing, $100 \mu \mathrm{L}$ of diluted sera was added and incubated overnight. After further washing, biotinylated mouse anti-human anti-DcR3 antibody was added $(2.0 \mu \mathrm{g} / \mathrm{mL})$ and incubated for $2 \mathrm{~h}$ at room temperature. Finally, streptavidin-HRP (R\&D Systems) was added for $30 \mathrm{~min}$. After removing HRP excess, $100 \mu \mathrm{L}$ of $1: 1$ mixture of $\mathrm{H}_{2} \mathrm{O}_{2}$ and tetramethylbenzidine ( $\mathrm{R} \& \mathrm{D}$ Systems) was added and absorbance was measured at $450 \mathrm{~nm}$ and corrected at $570 \mathrm{~nm}$ after $30 \mathrm{~min}$. Absorbance of each sample was plotted against a standard curve produced by serial dilutions of recombinant human DcR3. Concentrations were calculated via logarithmic analysis. All samples with an absorbance of less than the average of the zero standards + 2SD were considered as nondetectable. The lower limit of detection for the assay was $0.19 \mathrm{ng} / \mathrm{mL}$. To avoid interassay variability, in each separate assay, we run, in parallel, similar numbers of samples from patients and controls.

2.4. Statistical Analysis. Nonparametric tests were used for statistical analysis because of the highly skewed distribution of DcR3 levels associated with a great number of nondetectable values. In particular, comparison between groups was performed by Mann-Whitney (2 groups) or KruskalWallis (>2 groups) test. Values of DcR3 in the serum and ascites from the same patient were compared by Wilcoxon test. Categorical variables were compared by chi-squared test.
Spearman's $r$-test was used to assess correlations of DcR3 with other variables. Receiver operating characteristic (ROC) curve analysis was used to determine specificity, sensitivity, and optimal threshold values of sDCR3 (i.e., value offering the highest sum of sensitivity and specificity). In all cases, a $P$ value of $<0.05$ was considered to be significant.

\section{Results}

3.1. Patient Characteristics. The main patient- and diseaserelated characteristics are shown in Table 1. As expected, patients with $\mathrm{CVH}$ were younger than patients with $\mathrm{DC}$ and/or HCC. In the group of DC, there was an equal distribution of cases to CVH and alcoholic liver disease subgroups, whereas HCC cases were mostly related to $\mathrm{HBV}$ and $\mathrm{HCV}$ infections.

\subsection{Serum DcR3 Protein Concentration Is Elevated in Chronic} Liver Disease. A considerable proportion of controls (29\%) had serum DcR3 levels below the detection limit of the assay. This was also the case in $19 \%$ of patients with CVH. In contrast, all patients with either DC or HCC had detectable $\operatorname{sDcR} 3(P<0.001)$.

The levels of sDcR3 were significantly elevated in patients with chronic liver disease compared to controls (2.6 (0-63.8) versus $0.8(0-7.9) \mathrm{ng} / \mathrm{mL}$; median (range) $P<0.001)$ (Figure $1(\mathrm{a})$ ). When separate groups were compared, we observed a gradual increase in median (range) sDcR3 levels from controls $(0.8(0-7.9) \mathrm{ng} / \mathrm{mL})$ to 2.7 -fold average elevation in CVH patients $(1.1(0-4.2) \mathrm{ng} / \mathrm{mL}, P=0.006)$, an 8.8 -fold 


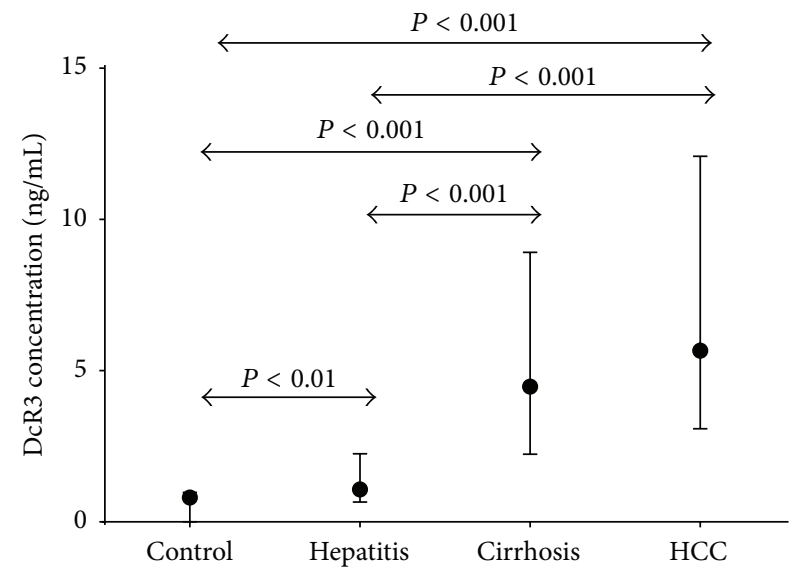

(a)

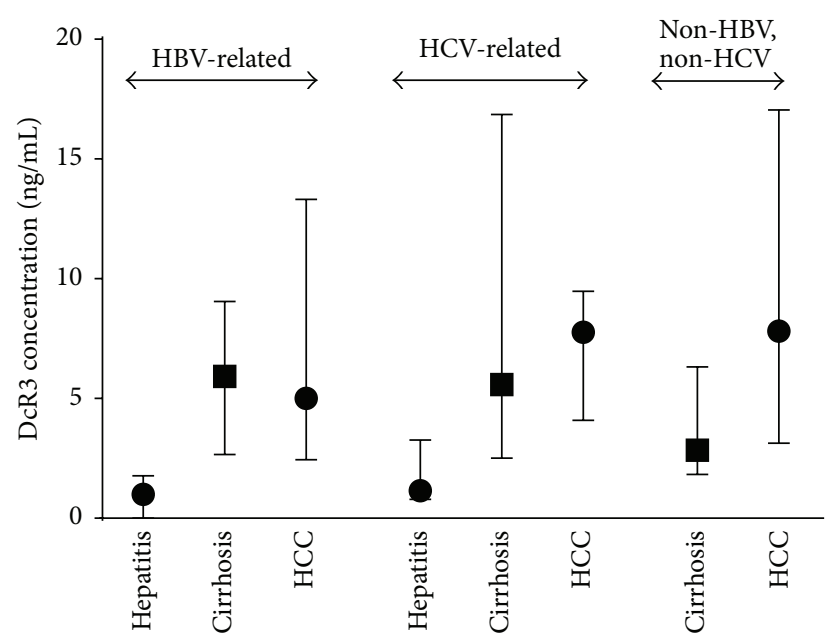

(b)

FIGURE 1: (a) DcR3 protein is highly elevated in the systemic circulation of patients with chronic liver disease. (b) sDcR3 levels are dependent upon the presence of cirrhosis and not the underlying cause of liver disease. Concentration of DcR3 in the sera was measured by ELISA. Values are expressed as median \pm interquartile range. Comparison between groups was performed by Mann-Whitney test.

increase in DC patients $(4.5(0.9-61.8) \mathrm{ng} / \mathrm{mL}, P<0.001)$, and, finally, a 10.2-fold increase in patients with HCC (5.7 (0.6-63.8) ng/mL, $P<0.001)$. There was also a significant difference in sDcR3 levels between patients with $\mathrm{CVH}$ and those with DC $(P<0.001)$ or $\operatorname{HCC}(P<0.001)$, while no difference was observed between patients with DC and HCC.

\subsection{Serum DcR3 Protein Concentration Correlates with Dis-} ease Progression and Fibrosis in Patients with Chronic Liver Disease. In patients with $\mathrm{CVH}$, there was no association between sDcR3 levels and age, sex, or viral genotype (for HCV-related cases). Furthermore, we observed that the concentration of sDcR3 in the serum was not associated with any specific etiology of the chronic liver disease. More specifically, when $\mathrm{HCV}$ and $\mathrm{HBV}$ infected patients were separately analyzed, the levels of sDcR3 were not associated with the underlying cause of liver disease but only with the presence of cirrhosis (Figure 1(b)). Similarly, when the subgroup of patients with cirrhosis was analyzed, no significant differences were observed among the different subgroups of patients with cirrhosis subdivided according to the etiology of liver disease (HBV, HCV, or alcohol related cirrhosis) (Figure 1(b)).

In regard to biochemical parameters, there was a trend towards higher sDcR3 values with increasing serum aminotransferases, but these correlations did not reach statistical significance $(P=0.085$ for AST and $P=0.053$ for ALT). In the 34 patients with a liver biopsy, we observed significant elevation of sDcR3 levels with worsening severity of fibrosis (stage). In particular, sDcR3 concentrations were higher in patients with fibrosis histological stage $\geq 4$ than that $\leq 3$ (median (range): $2.2(0.6-6.8)$ versus $0.9(0-5.1) \mathrm{ng} / \mathrm{mL}$, $P=0.003)$. Similarly, sDcR3 concentrations were higher in patients with severe fibrosis than in those without severe fibrosis by either liver biopsy (histological stage $\geq 4$ ) or elastography (stiffness $>10 \mathrm{kPa}$ ) (median (range): $2.2(0-$ 10.6) versus $1.2(0-5.9) \mathrm{ng} / \mathrm{mL}, P<0.001)$ or in those with compensated cirrhosis than in those without compensated cirrhosis (stage 5-6 or stiffness at elastography $>13 \mathrm{kPa})(3.5$ $(1.8-10.6)$ versus $1.2(0-6.5) \mathrm{ng} / \mathrm{mL}, P<0.001)$.

A trend for higher sDcR3 values was also observed in patients with moderate or severe necroinflammatory activity (histological grade $\geq 7$ ) than those with mild necroinflammatory activity at liver biopsy (grade $\leq 6)(1.4$ (0.9-5.7) versus $0.7(0-6.8) \mathrm{ng} / \mathrm{mL}, P=0.096)$. Interestingly, when only treatment-naive patients (in the subgroup of $\mathrm{CVH}$ ) were considered, sDcR3 concentrations were significantly higher in patients with moderate/severe inflammation than in those with mild inflammation $(1.7(0.9-3.7)$ versus $0.6(0-$ 5.0) ng/mL, $P=0.024$ ).

In patients with $\mathrm{DC}$, there was a positive correlation between sDcR3 levels and MELD scores (Spearman $r=$ 0.653, $P<0.001$ ) (Figure 2(a)). In addition, sDcR3 levels also correlated positively with other markers of severity of cirrhosis and particularly of hepatocellular synthesis, such as the international normalized ratio $(r=0.662, P<0.001)$ and serum bilirubin levels $(r=0.609, P<0.001)$ (Table 2$)$. All these correlations were independent of a specific liver disease etiology.

In the subgroup of 32 patients (which included patients with all types of liver disease but not established DC cases) who had undergone shear wave elastography, a strong positive correlation between sDcR3 levels and liver stiffness measurements was found (Spearman $r=0.698, P<0.001$ ) (Figure 2(b)).

Finally, as we inferred from the aforementioned findings a possible association of $s \mathrm{DcR} 3$ with fibrosis, we used receiver operating characteristic (ROC) curve analyses in order to determine the use of sDcR3 values for diagnosis of severe fibrosis or advanced liver disease. In $\mathrm{CVH}$ patients without 


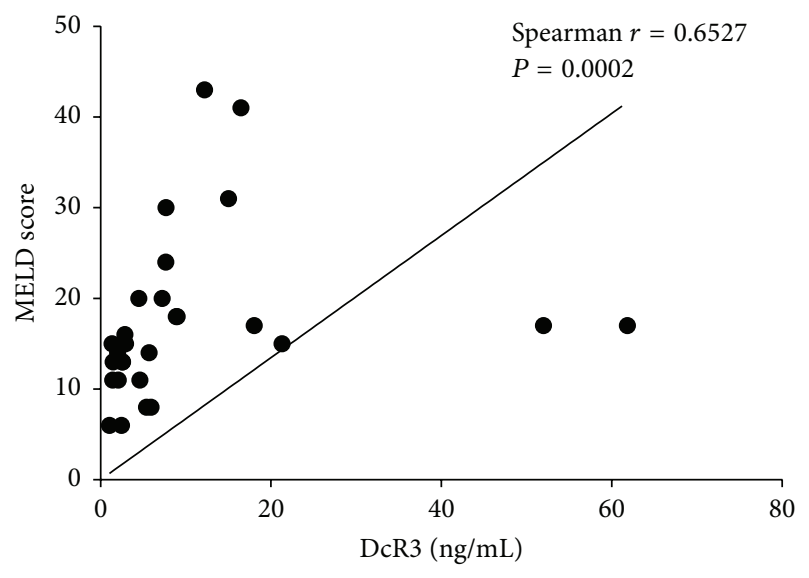

(a)

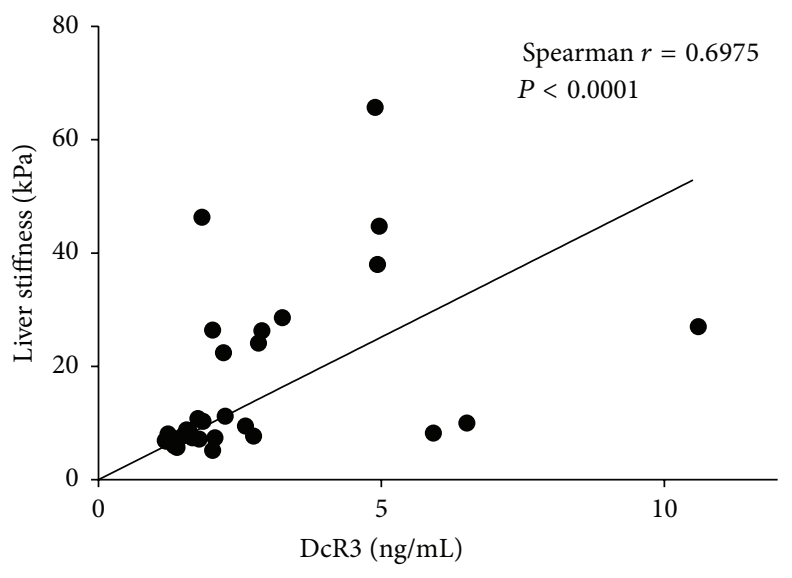

(b)

FIGURE 2: Serum concentration of DcR3 strongly correlates with markers of disease progression in patients with chronic liver disease. The serum concentration of DcR3 was measured by ELISA. Ishak scores (histological stage) were retrieved from the histological reports from patients with available liver biopsies. MELD scores were calculated for cirrhotic patients. The correlation of sDcR3 concentration with (a) the MELD score of patients with cirrhosis is shown; (b) the liver stiffness values of patients with chronic liver disease as measured by shear wave elastography. Statistical significance of correlation was calculated by use of Spearman's $r$-test.

TABLE 2: Correlation of sDcR3 with patient characteristics.

\begin{tabular}{lcc}
\hline \multicolumn{2}{c}{ Spearman correlation coefficient $(r)$} & $P$ \\
\hline Chronic viral hepatitis & \\
Age & 0.046 & NS \\
Sex & & NS \\
HCV genotype & & NS \\
AST & 0.331 & 0.085 \\
ALT & 0.369 & 0.053 \\
\hline DC & & \\
Age & 0.054 & NS \\
Sex & & NS \\
MELD score & 0.653 & $<0.001$ \\
AST & 0.299 & NS \\
ALT & 0.127 & NS \\
Bilirubin & 0.609 & $<0.001$ \\
INR & 0.662 & $<0.001$ \\
Creatinine & 0.243 & NS \\
\hline
\end{tabular}

NS: nonsignificant value.

DC, the area under the ROC curve for the diagnosis of severe fibrosis (histological stage $\geq 4$ or liver stiffness $>10 \mathrm{kPa}$ ) was $0.771(P=0.001)$ and the optimal cutoff value of sDcR3 levels was $1.82 \mathrm{ng} / \mathrm{mL}$ offering sensitivity of $83 \%$ and specificity of $80 \%$. When patients with DC were also included, the area under the ROC curve for the diagnosis of advanced liver disease was $0.852(P<0.001)$ and the optimal cutoff value of sDcR3 levels was $1.98 \mathrm{ng} / \mathrm{mL}$ offering sensitivity of $81 \%$ and specificity of $80 \%$.

Taken together, these results demonstrate that the presence of chronic liver disease is associated with significant elevations in circulating DcR3, with gradual increases observed with advanced stages of hepatic injury and more specifically with worsening fibrosis.

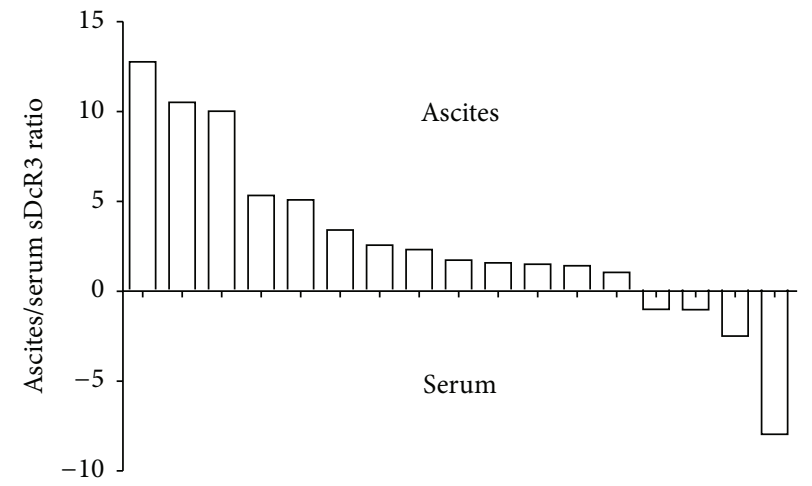

FIGURE 3: DcR3 protein is abundantly detected in ascitic fluid of cirrhotic patients. Serum and ascitic fluid were concomitantly obtained from individual patients with cirrhosis and the concentration of DcR3 was measured by ELISA in both. The ascites-to-serum ratio was determined. Each bar shown in the graph corresponds to a single individual.

3.4. DcR3 Protein Is Abundantly Detected in Ascitic Fluid of Cirrhotic Patients. DcR3 protein was always detected in ascitic fluid. To further establish a connection between DcR3 levels in ascites and liver dysfunction, we concomitantly calculated and compared the levels of sDcR3 protein in the ascitic fluid and the peripheral blood of individual patients. There was a correlation between the DcR3 levels in the ascitic fluid and the serum of patients with DC ( $r=0.417, P=0.09)$. In the vast majority of cases (88\%), the concentration of DcR3 in ascites far exceeded the respective value in the serum $(P=$ 0.013). This increase ranged from 150 tol270\% (Figure 3).

\section{Discussion}

In the current study, we report that circulating DcR3 protein is elevated in patients with chronic liver disease and may serve 
as an indicator for disease progression, such as development of fibrosis during chronic hepatitis and functional deterioration in cirrhosis.

Several findings support this concept. First, patients with DC had significantly higher concentrations of DcR3 in comparison to patients with $\mathrm{CVH}$. While it is true that patients with $\mathrm{CVH}$ were younger than patients with DC and/or HCC in our cohort, this difference did not have any influence on data analysis as sDcR3 concentration has been shown to not be affected by age in previous studies $[10,19]$. We observed that a considerable proportion of patients in the CVH group did not have detectable DcR3 in the serum, resembling the situation in $\mathrm{HC}$. In contrast, $\mathrm{sDcR} 3$ was always present in the serum of patients with decompensated cirrhosis. This indicates that as soon as decompensated cirrhosis is established, secretion of DcR3 becomes a stable phenotype. Second, we observed that the association between cirrhosis or increased liver stiffness in SWE and elevated DcR3 occurred irrespectively of the specific etiology of chronic liver disease. This indicates that it is not a specific causative agent (HBV, $\mathrm{HCV}$, and alcohol) that drives the elevation of sDcR3, but the inflammatory/fibrotic course per se. These findings are in line with previous work by Kim et al. who reported that increased immunolocalization of DcR3 protein and detection of DcR3-specific mRNA sequences in chronic HCV-related liver disease were mainly seen in areas with prominent fibrosis [20]. Interestingly, two recent studies may support this possible role of DcR3 in the inflammatory/fibrotic pathway. It was reported that DcR3 concentrations were increased in patients with primary biliary cirrhosis in both local and systemic levels when compared to healthy controls [21], while researchers from Japan suggest that sDcR3 values may be helpful in differentiating between active and inactive chronic hepatitis B in the subgroup of hepatitis B e antigennegative patients [22]. Furthermore, in our study, within the population of patients with chronic hepatitis, the highest DcR3 values were seen in those with advanced Ishak scores in liver biopsy (i.e., with increasing levels of fibrosis). Although the number of patients in the cirrhosis subgroup (Ishak 56) was small, the gradual increase in sDcR3 concentration with increasing presence of fibrosis confirms the previously shown association between DcR3 and cirrhosis. Finally, sDcR3 concentrations were observed to have a strong positive correlation with the presence of liver fibrosis as assessed by SWE liver stiffness measurements.

As DcR3 exists solely in a soluble form, it is possible that the elevated sDcR3 content shown in our study originates in regions with cirrhotic transformation of the liver. The hepatic derivation of sDcR3 is also supported by our paired examinations which showed that DcR3 values in ascitic fluid far exceeded the corresponding values in the serum of the same patient. Further supporting this hypothesis are the results from a previous study, which demonstrated that, in patients with HCC, the serum concentration of DcR3 directly correlated with the presence of paracirrhosis [14]. Interestingly, our group is the first to report expression of the DcR3 protein in the ascitic fluid obtained from patients with liver cirrhosis as previously this cytokine had been only studied in ascitic samples from patients with epithelial ovarian cancer [23].

DcR3 acts as decoy-receptor for FasL and LIGHT, blocking apoptotic signals induced by these proteins [24-26]. Thus, previous research has focused on the potential role of DcR3 in carcinogenesis in a variety of neoplastic tissues in general and more specifically in the liver. Indeed, several studies have shown increased local and systemic expression of DcR3 in patients with HCC $[7,14,15,27]$. Moreover, DcR3 was shown to protect hepatoma cells against apoptosis induced by FasL [28]. Differently from these reports, in our present study, we comparatively examined the sDcR3 protein content along the various steps of chronic liver disease. We were able to clearly demonstrate that elevation of sDcR3 is not specific for the presence of neoplastic hepatic transformation; rather, it occurs at an earlier step, as DcR3 is already present in high levels in the systemic circulation when cirrhosis is established. Longitudinal serial measurements of sDcR3 in individual patients will be required in order to better depict the temporal association between significant DcR3 elevations and transition to cirrhosis and HCC. This might be of significant clinical importance as reliable soluble markers of disease progression and fibrosis during chronic liver disease are currently lacking.

A second present finding with potential importance is the strong positive correlation between elevated serum DcR3 and progressive liver disease. Very interestingly, such an association was evident in all patient subgroups. In particular, patients with CVH and high sDcR3 levels had increased probability for advanced liver fibrosis stage, as indicated by higher Ishak and liver stiffness scores. Similarly, in the cirrhotic population, sDcR3 positively correlated with high MELD and SWE scoring, markers of functional deterioration, and the extent of liver fibrosis, respectively. Notably, a similar association with adverse outcomes appears to take place in HCC also, as it was shown that higher local or systemic DcR3 expression in this population was associated with advanced TNM stage or increased incidence of local invasion or distant metastasis [14]. A similar positive correlation of elevated DcR3 expression with unfavorable disease course was also noted not only in other neoplasms $[7,8]$ but also in nonneoplastic conditions [10-13]. Taken together, these results indicate that finding a highly elevated DcR3 concentration in patients with chronic inflammatory or neoplastic conditions, including chronic liver disease, may be considered a poor prognostic factor. The biological explanation for this phenomenon is unclear but given the decoy/suppressive nature of DcR3, its excessive secretion may represent a compensatory reflex to initial upregulation of its ligands (FasL, LIGHT, and TL1A), which may occur as part of the pathogenetic disease process. Alternative explanations may involve a pro-tumor-growth result of the immunosuppressive function of DcR3. Indeed, DcR3 has been shown to favor the polarization of macrophages towards an M2 phenotype, which is characterized by decreased phagocytic activity [29]. In addition, DcR3 treated macrophages adapt to the phenotype of tumor-associated macrophages, offering therefore a survival advantage to tumor cells [30]. In all, it can be speculated that the elevated DcR3 may be pathogenetically 
involved in cancer progression and not merely a surrogate marker of aggressive disease course.

\section{Conclusions}

Our present findings support the hypothesis that serum DcR3 is gradually elevated during chronic liver disease and reaches its peak level after fibrosis is established. In addition, DcR3 may serve as a reliable marker of progressive disease. Further studies in larger and better selected patient populations are needed to substantiate the importance of this marker in the clinical management of patients with chronic liver conditions.

\section{Competing Interests}

The authors declare that they have no competing interests.

\section{Acknowledgments}

This study has been cofinanced by the European Union (European Social Fund, ESF) and Greek national funds through the Operational Program "Education and Lifelong Learning" of the National Strategic Reference Framework (NSRF), Research Funding Program, Heracleitus II: Investing in Knowledge Society through the European Social Fund, of which Dr. Garyfallia Kaltsa was a recipient.

\section{References}

[1] A. Forner, J. M. Llovet, and J. Bruix, "Hepatocellular carcinoma," The Lancet, vol. 379, no. 9822, pp. 1245-1255, 2012.

[2] G. Szabo and D. Lippai, "Molecular hepatic carcinogenesis: impact of inflammation," Digestive Diseases, vol. 30, no. 3, pp. 243-248, 2012.

[3] W.-W. Lin and S.-L. Hsieh, "Decoy receptor 3: a pleiotropic immunomodulator and biomarker for inflammatory diseases, autoimmune diseases and cancer," Biochemical Pharmacology, vol. 81, no. 7, pp. 838-847, 2011.

[4] C. Zhan, Y. Patskovsky, Q. Yan et al., "Decoy strategies: the structure of TL1A:DcR3 complex," Structure, vol. 19, no. 2, pp. 162-171, 2011.

[5] G. Bamias, K. Evangelou, T. Vergou et al., "Upregulation and nuclear localization of TNF-like Cytokine 1A (TL1A) and its receptors DR3 and DcR3 in psoriatic skin lesions," Experimental Dermatology, vol. 20, no. 9, pp. 725-731, 2011.

[6] C. Bai, B. Connolly, M. L. Metzker et al., "Overexpression of M68/DcR3 in human gastrointestinal tract tumors independent of gene amplification and its location in a four-gene cluster," Proceedings of the National Academy of Sciences of the United States of America, vol. 97, no. 3, pp. 1230-1235, 2000.

[7] Y. Wu, B. Han, H. Sheng et al., "Clinical significance of detecting elevated serum DcR3/TR6/M68 in malignant tumor patients," International Journal of Cancer, vol. 105, no. 5, pp. 724-732, 2003.

[8] Z. Ge, A. J. Sanders, L. Ye, Y. Wang, and W. G. Jiang, "Expression of death decoy receptor-3 (DcR3) in human breast cancer and its functional effects on breast cancer cells in vitro," Journal of Experimental Therapeutics and Oncology, vol. 9, no. 2, pp. 109118, 2011.
[9] J. Chen, L. Zhang, and S. Kim, "Quantification and detection of DcR3, a decoy receptor in TNFR family," Journal of Immunological Methods, vol. 285, no. 1, pp. 63-70, 2004.

[10] G. Bamias, G. Kaltsa, S. I. Siakavellas et al., "Differential expression of the TL1A/DcR3 system of TNF/TNFR-like proteins in large vs. small intestinal Crohn's disease," Digestive and Liver Disease, vol. 44, no. 1, pp. 30-36, 2012.

[11] G. Bamias, G. Kaltsa, S. I. Siakavellas et al., "High intestinal and systemic levels of decoy receptor 3 (DcR3) and its ligand TL1A in active ulcerative colitis," Clinical Immunology, vol. 137, no. 2, pp. 242-249, 2010.

[12] G. Bamias, S. I. Siakavellas, K. S. Stamatelopoulos, E. Chryssochoou, C. Papamichael, and P. P. Sfikakis, "Circulating levels of TNF-like cytokine 1A (TL1A) and its decoy receptor 3 (DcR3) in rheumatoid arthritis," Clinical Immunology, vol. 129, no. 2, pp. 249-255, 2008.

[13] S. Kim, L. Mi, and L. Zhang, "Specific elevation of DcR3 in sera of sepsis patients and its potential role as a clinically important biomarker of sepsis," Diagnostic Microbiology and Infectious Disease, vol. 73, no. 4, pp. 312-317, 2012.

[14] M. Yang, G. Chen, Y. Dang, and D. Luo, "Significance of decoy receptor 3 in sera of hepatocellular carcinoma patients," Upsala Journal of Medical Sciences, vol. 115, no. 4, pp. 232-237, 2010.

[15] G. Chen and D. Luo, "Expression of decoy receptor 3 in liver tissue microarrays," National Medical Journal of India, vol. 21, no. 6, pp. 275-278, 2008.

[16] C. Chen, C. Zhang, G. Zhuang et al., "Decoy receptor 3 overexpression and immunologic tolerance in hepatocellular carcinoma (HCC) development," Cancer Investigation, vol. 26, no. 10, pp. 965-974, 2008.

[17] European Association for the Study of the Liver and European Organisation for Research and Treatment of Cancer, "EASLEORTC clinical practice guidelines: management of hepatocellular carcinoma," Journal of Hepatology, vol. 56, no. 4, pp. 908943, 2012.

[18] K. Ishak, A. Baptista, L. Bianchi et al., "Histological grading and staging of chronic hepatitis," Journal of Hepatology, vol. 22, no. 6, pp. 696-699, 1995.

[19] S.-C. Weng, K.-H. Shu, M.-J. Wu et al., "Expression of decoy receptor 3 in kidneys is associated with allograft survival after kidney transplant rejection," Scientific Reports, vol. 5, article 12769, 2015.

[20] S. Kim, V. Kotoula, P. Hytiroglou, D. Zardavas, and L. Zhang, "Significance of increased expression of decoy receptor 3 in chronic liver disease," Digestive and Liver Disease, vol. 41, no. 8, pp. 591-598, 2009.

[21] Y. Aiba, K. Harada, A. Komori et al., "Systemic and local expression levels of TNF-like ligand 1A and its decoy receptor 3 are increased in primary biliary cirrhosis," Liver International, vol. 34, no. 5, pp. 679-688, 2014.

[22] Y. Hou, P. Xu, X. Lou et al., "Serum decoy receptor 3 is a useful predictor for the active status of chronic hepatitis $b$ in hepatitis b e antigen-negative patients," Tohoku Journal of Experimental Medicine, vol. 230, no. 4, pp. 227-232, 2013.

[23] J. P. Connor and M. Felder, "Ascites from epithelial ovarian cancer contain high levels of functional decoy receptor 3 (DcR3) and is associated with platinum resistance," Gynecologic Oncology, vol. 111, no. 2, pp. 330-335, 2008.

[24] R. M. Gill and J. S. Hunt, "Soluble receptor (DcR3) and cellular inhibitor of apoptosis-2 (cIAP-2) protect human cytotrophoblast cells against LIGHT-mediated apoptosis," American Journal of Pathology, vol. 165, no. 1, pp. 309-317, 2004. 
[25] B. Han and J. Wu, "DcR3 protects islet $\beta$ cells from apoptosis through modulating Adcyapl and Bank1 expression," The Journal of Immunology, vol. 183, no. 12, pp. 8157-8166, 2009.

[26] W. Li, C. Zhang, C. Chen, and G. Zhuang, "Correlation between expression of DcR3 on tumor cells and sensitivity to FasL," Cellular \& Molecular Immunology, vol. 4, no. 6, pp. 455-460, 2007.

[27] V. J. Wroblewski, D. R. Witcher, G. W. Becker et al., "Decoy receptor 3 (DcR3) is proteolytically processed to a metabolic fragment having differential activities against Fas ligand and LIGHT,' Biochemical Pharmacology, vol. 65, no. 4, pp. 657-667, 2003.

[28] H.-W. Shen, S.-L. Gao, Y.-L. Wu, and S.-Y. Peng, "Overexpression of decoy receptor 3 in hepatocellular carcinoma and its association with resistance to Fas ligand-mediated apoptosis," World Journal of Gastroenterology, vol. 11, no. 38, pp. 5926-5930, 2005.

[29] Y. C. Chang, T. L. Hsu, H. H. Lin et al., "Modulation of macrophage differentiation and activation by decoy receptor 3," Journal of Leukocyte Biology, vol. 75, pp. 486-494, 2004.

[30] S.-K. Tai, H.-C. Chang, K.-L. Lan et al., "Decoy receptor 3 enhances tumor progression via induction of tumor-associated macrophages," Journal of Immunology, vol. 188, no. 5, pp. 24642471, 2012. 


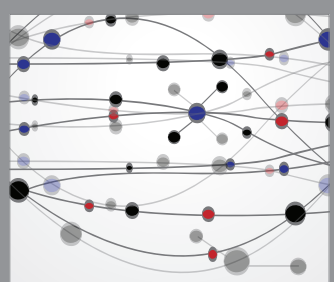

The Scientific World Journal
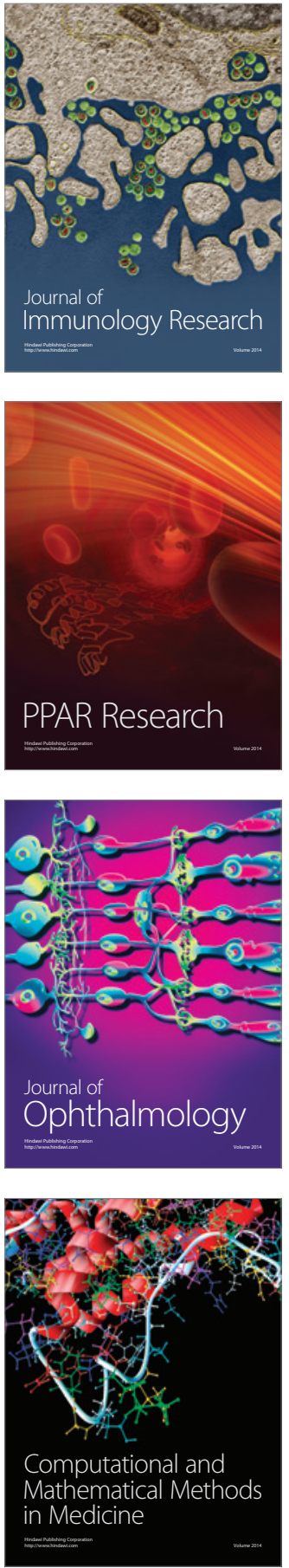

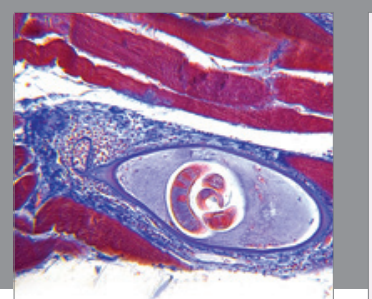

Gastroenterology Research and Practice

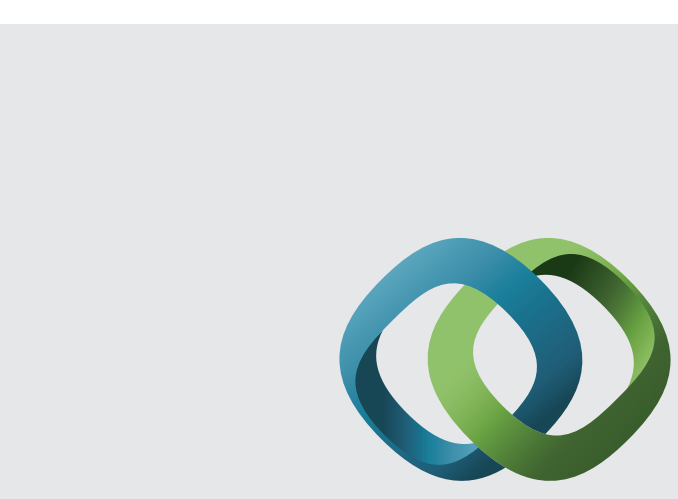

\section{Hindawi}

Submit your manuscripts at

http://www.hindawi.com
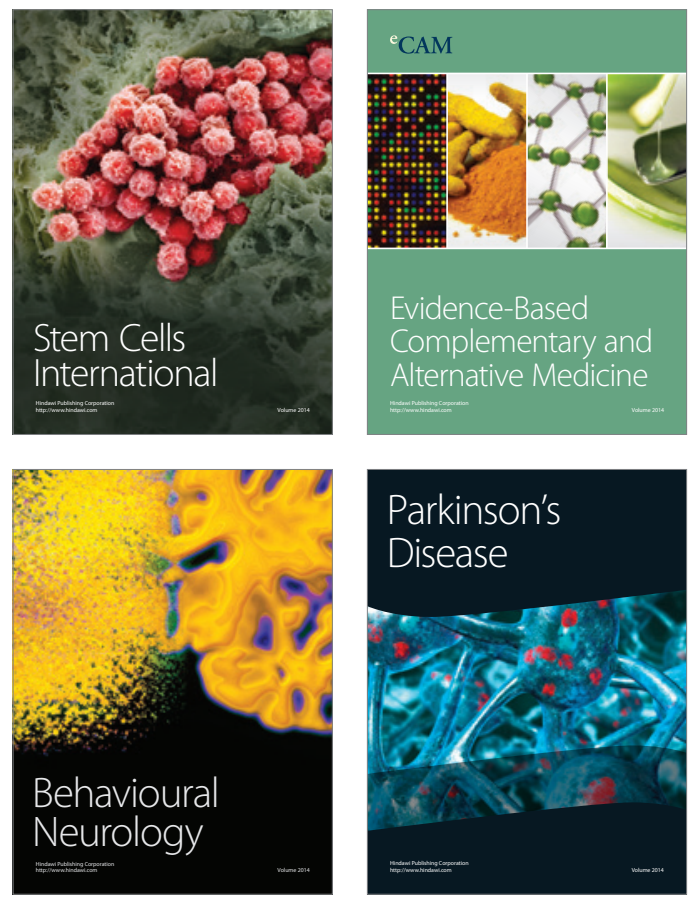
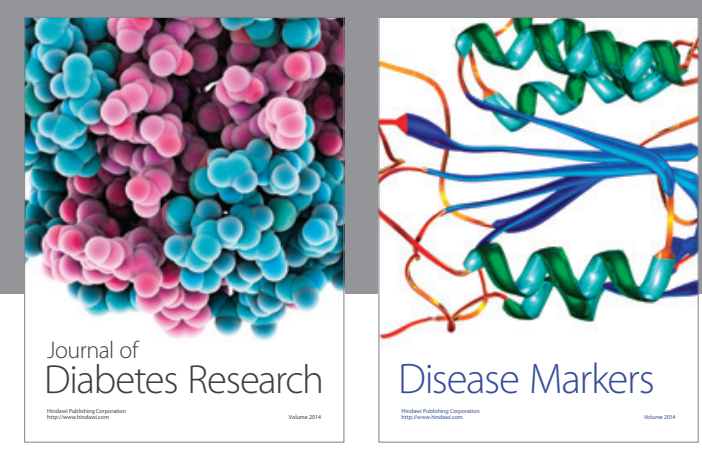

Disease Markers
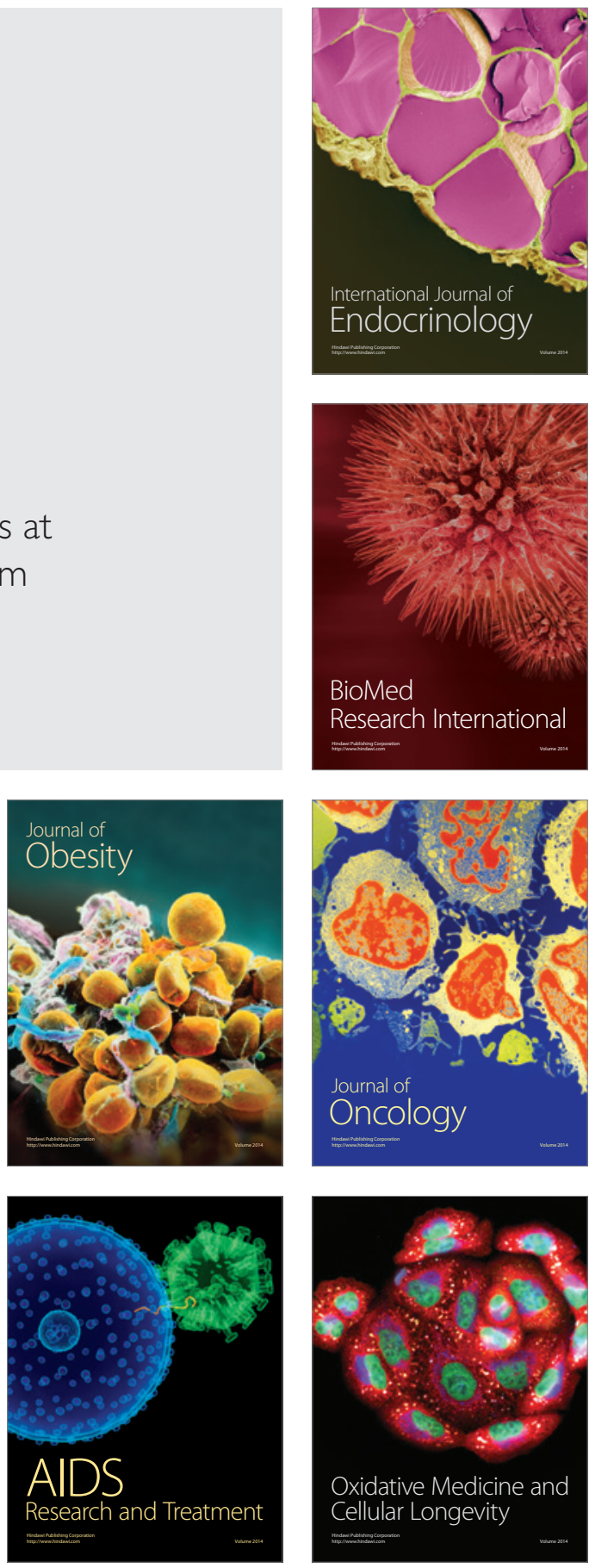